\title{
Localized multibunch modes in accelerators
}

\author{
G. Dugan \\ Laboratory of Nuclear Studies, Cornell University, Ithaca, New York 14853
}

(Received 25 November 1998; published 29 September 1999)

\begin{abstract}
This paper will explore the possibility of the formation of localized modes in the coupled transverse oscillations of bunches in an accelerator. Such modes are characterized by a large amplitude excitation of one of the bunches, with little or no excitation of the other bunches. They are very similar to intrinsic localized modes predicted to appear in lattice excitations of anharmonic condensed matter systems. The existence of the phenomenon in accelerators requires long-range coupling between the bunches, and quartic terms in the effective potential experienced by the bunches. The discussion in this paper considers the specific cases of a long-range coupling due to the resistive wall impedance, and nonlinearities in the transverse force caused by octupoles. The general equations for the localized mode frequencies and amplitudes are derived. The equations are applied to the specific cases of 9 and 21 equally spaced, equally populated bunches in the Cornell electron storage ring. For both cases, with the currently available octupole strength, and with currents of $8 \mathrm{~mA}$ per bunch, localized modes with amplitudes of about $2 \mathrm{~mm}$, and with frequency shifts of 50-100 s $\mathrm{s}^{-1}$ relative to the linear coupled-bunch mode frequencies, may be possible.
\end{abstract}

PACS numbers: 29.27.Bd, 41.85.-p, 63.20.Pw

\section{INTRODUCTION}

It has been suggested [1,2] that the phenomenon of intrinsic localized modes in anharmonic condensed matter systems [3-7] may also be observed in the excitations of multibunch modes in accelerators, in the presence of significant lattice nonlinearities. In this paper, this possibility is explored quantitatively, for multibunch coupling produced by the resistive wall impedance, and an octupole-generated nonlinearity.

The basic physical mechanism involved in the development of localized modes is quite straightforward. In a machine with many bunches, every bunch will be coupled to the other bunches through the machine impedance. This coupling results in a spectrum of normal modes, with some spread in the normal mode frequencies. Because of the coupling, transverse excitation of a single bunch will eventually result in oscillation amplitudes for all the bunches. However, in the presence of an octupole, the oscillation frequency for a bunch will be a function of its oscillation amplitude. If the frequency shift due to the oscillation amplitude of a single bunch is substantially larger than the frequency spread of the linear normal modes, this bunch no longer couples to the others, and the oscillation will remain "localized" on a single bunch. This type of behavior is referred to as a localized mode.

The standard formalism for the treatment of coupled bunch oscillations forms the starting point for the analysis. The normal mode frequencies are specified in terms of a general effective transverse impedance. The impedance is taken to be that due to the resistive wall. As specific examples, the normal mode spectrum is displayed for 9 and 21 equally spaced, equally populated bunches in the Cornell electron storage ring (CESR). An octupole is then introduced, which produces a dependence of the tune on amplitude, makes the coupled bunch equations nonlinear, and allows for the possibility of localized modes. A Green's function approach is developed to allow an iterative solution to the nonlinear coupled bunch equations. The equations which exhibit the localized modes are developed using this Green's function. These equations give the frequency shift (relative to the linear coupled bunch frequencies) and the mode pattern for the localized modes. The equations are applied to the cases of $2 \mathrm{~mm}$ amplitude excitations of 9 and 21 bunches in CESR, using the parameters corresponding to the existing octupoles in the machine. As a check on the approximations used in the analytical treatment, a numerical simulation of the localized mode evolution is also carried out.

\section{LINEAR NORMAL MODES}

Consider $M$ equally spaced bunches in a ring, of equal population. Let $y_{n}(t)(n=0,1, \ldots, M-1)$ be the "snapshot" transverse (vertical) displacement of the $n$th bunch. The displacement is given by

$$
y_{n}(t)=\tilde{y}_{n} \exp (-i \Omega t),
$$

where $\tilde{y}_{n}$ (a complex number) represents the amplitude and phase of bunch $n$ at time $t=0$.

The equation of motion [8] for bunch $n$, in the rigidbeam approximation is

$$
\frac{d^{2} y_{n}(t)}{d t^{2}}+\omega_{\beta}^{2} y_{n}(t)=-\frac{N r_{0} c}{\gamma T_{0}} \sum_{k=0}^{\infty} \sum_{m=0}^{M-1} W_{1}\left(-k C-\frac{m-n}{M} C\right) y_{m}\left(t-k T_{0}-\frac{m-n}{M} T_{0}\right) .
$$


In this equation, $W_{1}(z)$ is the transverse dipole wake function, $\omega_{\beta}=\nu \omega_{0}$ is the vertical betatron frequency, $N$ is the number of particles per bunch, $C$ is the ring circumference, $T_{0}=\frac{c}{C}=\frac{2 \pi}{\omega_{0}}$ is the revolution period, and $\gamma=\frac{E}{m_{0} c^{2}}$. Using the above form for $y_{n}(t)$, we can write Eq. (2) (for $\Omega \sim \omega_{\beta}$ ) as

$$
\frac{d^{2} y_{n}(t)}{d t^{2}}+\omega_{\beta}^{2} y_{n}(t)=-\frac{N r_{0} c}{\gamma T_{0}} \sum_{k=0}^{\infty} \sum_{m=0}^{M-1} W_{1}\left(-k C-\frac{m-n}{M} C\right) \exp \left[i \omega_{\beta} T_{0}\left(k+\frac{m-n}{M}\right)\right] y_{m}(t) .
$$

Transforming to the frequency domain and introducing the transverse impedance $Z^{\perp}(\omega)$, we have

$$
\frac{d^{2} y_{n}(t)}{d t^{2}}+\omega_{\beta}^{2} y_{n}(t)-\sum_{m=0}^{M-1} y_{m}(t) L(m-n)=0,
$$

with

$$
L(m-n)=i \frac{4 \pi N r_{0}}{\mu_{0} c \gamma T_{0}^{2}} \sum_{p=-\infty}^{\infty} Z^{\perp}\left(\omega_{\beta}+p \omega_{0}\right) \exp \left(-2 \pi i p \frac{m-n}{M}\right)
$$

The normal modes of the $M$ bunches are obtained by the usual technique. Let the normal modes $q_{n}$ given in terms of $y_{n}$ by the relation

$$
\mathbf{q}=\overleftrightarrow{\mathbf{C}} \cdot \mathbf{y}
$$

in which $\overleftrightarrow{\mathbf{C}}$ is a matrix. In matrix form, the equation of motion (4) is

$$
\ddot{\mathbf{y}}+\overleftrightarrow{\mathbf{S}} \cdot \mathbf{y}=0
$$

in which

$$
S_{m n}=\omega_{\beta}^{2} \delta_{m n}-L(n-m) .
$$

Using Eq. (6) to introduce the normal modes, this becomes

$$
\ddot{\mathbf{q}}+\overleftrightarrow{\mathbf{C}} \cdot \overleftrightarrow{\mathbf{S}} \cdot \overleftrightarrow{\mathbf{C}}-1 \mathbf{q}=0
$$

The matrix $\overleftrightarrow{\mathbf{C}}$ is required to diagonalize $\overleftrightarrow{\mathbf{S}}$. The eigenvalues are the normal mode frequencies. The required matrix is

$$
C_{l n}=\frac{1}{\sqrt{M}} \exp \left(\frac{-2 \pi i l n}{M}\right) .
$$

The matrix $\overleftrightarrow{\mathbf{C}}$ obeys the following orthonormality condition:

$$
\sum_{\alpha=0}^{M-1} C_{l \alpha} C_{\alpha n}^{*}=\frac{1}{M} \sum_{\alpha=0}^{M-1} \exp \left(\frac{2 \pi i \alpha(n-l)}{M}\right)=\delta_{n-l, r M},
$$

in which $r$ is any integer. Since $C_{l n}^{*}=C_{n l}^{*}=C_{n l}^{-1}, \overleftrightarrow{\mathbf{C}}$ is a unitary matrix. Using Eq. (11), it follows that the eigenvalues are

$$
\Omega_{l}^{2}=\omega_{\beta}^{2}-i \frac{4 \pi M N r_{0}}{\mu_{0} c \gamma T_{0}^{2}} \sum_{r=-\infty}^{\infty} Z^{\perp}\left(\omega_{\beta}+(r M+l) \omega_{0}\right),
$$

in which $l$ is the normal mode index. This relation between the mode frequency and the mode number is the analog of the $\omega(k)$ dispersion relation encountered in condensed matter systems. The relation between the displacement of the $n$th bunch and the normal modes $q_{l}$ is given by inverting Eq. (6), using Eq. (11),

$$
y_{n}(t)=\sum_{l=0}^{M-1} C_{n l}^{-1} q_{l}(t)=\frac{1}{\sqrt{M}} \sum_{l=0}^{M-1} \exp \left(\frac{2 \pi i l n}{M}\right) q_{l}(t) .
$$

We can define the frequency shift of normal mode $l$ using the approximation (for $\Omega_{l} \approx \omega_{\beta}$ )

$$
\Omega_{l}^{2}-\omega_{\beta}^{2}=\left(\Omega_{l}-\omega_{\beta}\right)\left(\Omega_{l}+\omega_{\beta}\right) \approx 2 \Delta \Omega_{l} \omega_{\beta} .
$$

So we have

$$
\Delta \Omega_{l}=-i \frac{2 \pi M N r_{0}}{\omega_{\beta} \mu_{0} c \gamma T_{0}^{2}} \sum_{r=-\infty}^{\infty} Z^{\perp}\left(\omega_{\beta}+(r M+l) \omega_{0}\right) .
$$

The above expression is correct only for a point bunch, with zero chromaticity. To include the effects of a finite bunch length (assumed Gaussian, of width $\sigma_{z}$ ) and a finite chromaticity $\xi$, we make the replacement [9]

$$
\sum_{r=-\infty}^{\infty} Z^{\perp}\left(\omega_{\beta}+(r M+l) \omega_{0}\right) \rightarrow \frac{c T_{0}}{2 M \sigma_{z} \sqrt{\pi}}\left(Z_{l}^{\perp}\right)_{\mathrm{eff}},
$$

with

$$
\left(Z_{l}^{\perp}\right)_{\mathrm{eff}}=\frac{\sum_{r=-\infty}^{\infty} Z^{\perp}\left(\omega_{r, l}\right) W\left(\omega_{r, l}, \omega_{\xi}, \sigma_{z}\right)}{\sum_{r=-\infty}^{\infty} W\left(\omega_{r, l}, \omega_{\xi}, \sigma_{z}\right)} .
$$

In this expression,

$$
\begin{aligned}
W\left(a, b, \sigma_{z}\right) & =\exp \left(-\frac{(a-b)^{2} \sigma_{z}^{2}}{c^{2}}\right), \\
\omega_{r, l} & =\omega_{\beta}+(r M+l) \omega_{0}, \\
\omega_{\xi} & =\frac{\xi \omega_{\beta}}{\eta} .
\end{aligned}
$$

So we get

$$
\Delta \Omega_{l}=-i \frac{\sqrt{\pi} N r_{0}}{\omega_{\beta} \mu_{0} \gamma \sigma_{z} T_{0}}\left(Z_{l}^{\perp}\right)_{\mathrm{eff}} .
$$




\section{COUPLING IMPEDANCE}

We will consider only the resistive wall impedance, for the sake of simplicity. The existence and general character of the localized modes is not expected to depend sensitively on the details of the frequency dependence of the impedance.

The transverse impedance associated with the resistive wall [10] is, for low frequencies $\omega \ll\left(\frac{\mu_{0} \sigma}{4 \pi} \frac{c^{4}}{b^{2}}\right)^{1 / 3}$,

$$
Z^{\perp}(\omega)=\frac{Z_{0} C}{4 \pi b^{3}} \sqrt{\frac{8}{\mu_{0} \sigma}}|\omega|^{1 / 2} \frac{[1-\operatorname{sgn}(\omega) i]}{\omega} .
$$

In this equation, $\sigma$ is the conductivity of the vacuum chamber wall, $b$ is the radius of the (assumed round) vacuum chamber, and $Z_{0}$ is the impedance of free space $(377 \Omega)$.

For the resistive wall, then, we have, combining Eqs. (19) and (20),

$$
\Delta \Omega_{l}=-i \frac{N r_{0}}{4 \sqrt{\pi} \omega_{\beta} \mu_{0} \gamma T_{0}^{2}} \frac{Z_{0}}{\sigma_{z} b^{3}} \sqrt{\frac{8}{\mu_{0} \sigma}} \frac{\sum_{r=-\infty}^{\infty}\left\{\left|\omega_{r, l}\right|^{1 / 2} \frac{\left[1-\operatorname{sgn}\left(\omega_{r, l}\right) i\right]}{\omega_{r, l}} W\left(\omega_{r, l}, \omega_{\xi}, \sigma_{z}\right)\right\}}{\sum_{r=-\infty}^{\infty} W\left(\omega_{r, l}, \omega_{\xi}, \sigma_{z}\right)} .
$$

\section{SPECTRUM OF THE NORMAL MODES}

We choose the following specific case to calculate the normal mode spectrum for CESR. We take $N=1.3 \times$ $10^{11}$ (corresponding to about $8 \mathrm{~mA}$ per bunch), $\nu=9.61$, and $C=778 \mathrm{~m}$. We take an aluminum $[\sigma=3.5 \times$ $\left.10^{7}\left(\Omega \mathrm{m}^{-1}\right)\right]$ vacuum chamber of radius $b=25 \mathrm{~mm}$. We take $\eta=0.01$, and a bunch length of $\sigma_{z}=20 \mathrm{~mm}$. The chromaticity is set to $\xi=2$, which makes all of the multibunch modes stable (i.e., they have a negative imaginary part). Figures 1 and 2 give the real and imaginary parts of the frequency shift [Eq. (21)], as a function of the mode number, for $M=21$ bunches.

Figure 3 shows the frequencies at which the various modes would be observed on a spectrum analyzer.

Figures 4-6 present the same information, but for $M=9$ bunches.

\section{OCTUPOLE NONLINEARITY}

We introduce an octupole into the ring at the location $s_{1}$. Provided that we are not operating close to a second-order or fourth-order resonance, the octupole field perturbation results primarily in a dependence of the betatron tune, $\nu=\frac{\omega_{\beta}}{\omega_{0}}$, on amplitude,

$$
\nu(a)=\nu+\mu a^{2},
$$

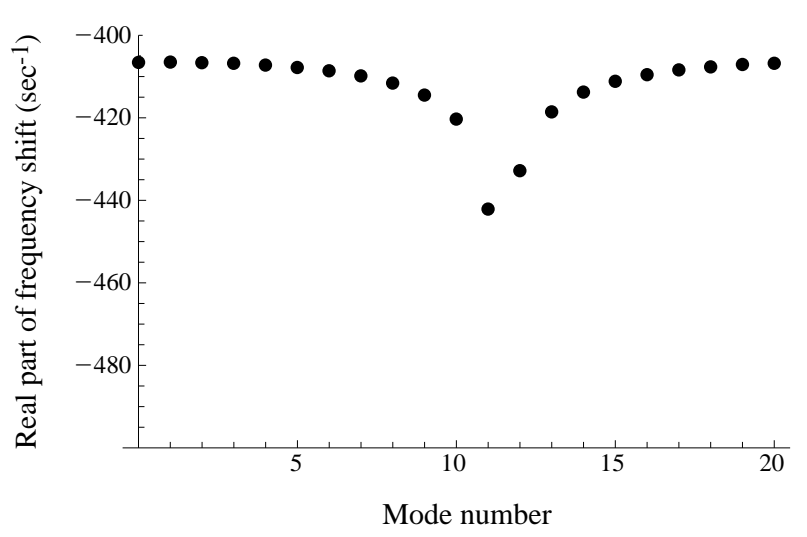

FIG. 1. The real part of $\Delta \Omega_{\mu}$ vs mode number $\mu$, for $M=21$. in which

$$
\mu=\frac{3}{16 \pi} \frac{k_{3} L}{6} \frac{\beta\left(s_{1}\right)^{2}}{\beta_{0}} .
$$

In this equation, $k_{3}=\frac{1}{B \rho} \frac{d^{3} B}{d y^{3}}$ is the octupole strength, $L$ is the octupole length, $\beta\left(s_{1}\right)$ is the beta function at the location of the octupole, and $\beta_{0}$ is the beta function at the point at which the oscillation amplitude is $a$.

\section{DEVELOPMENT OF GREEN'S FUNCTION}

To solve the nonlinear equations which result from the inclusion of the octupole, we will use the techniques discussed in Refs. [3-5]. This involves the development of Green's function for the equation of motion, Eq. (4). In that equation, if we let $y_{n}(t)=\tilde{y}_{n} \exp (-i \Omega t)$, we have

$$
-\Omega^{2} \tilde{y}_{n}+\omega_{\beta}^{2} \tilde{y}_{n}-\sum_{m=0}^{M-1} \tilde{y}_{m} L(m-n)=0 .
$$

If we define the matrix

$$
R_{m n}(\Omega)=\left(-\Omega^{2}+\omega_{\beta}^{2}\right) \delta_{m n}-L(n-m),
$$

then the equation of motion is

$$
\sum_{m=0}^{M-1} R_{n m}(\Omega) \tilde{y}_{m}=0
$$

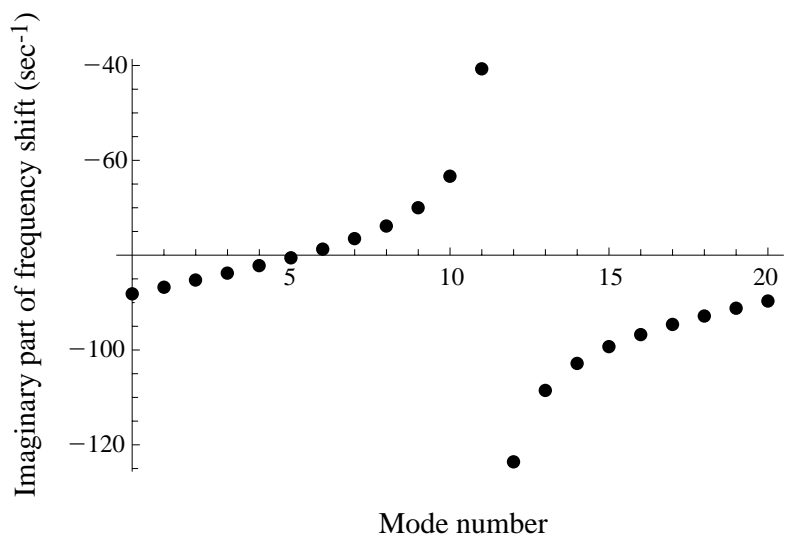

FIG. 2. The imaginary part of $\Delta \Omega_{\mu}$ vs mode number $\mu$, for $M=21$. 


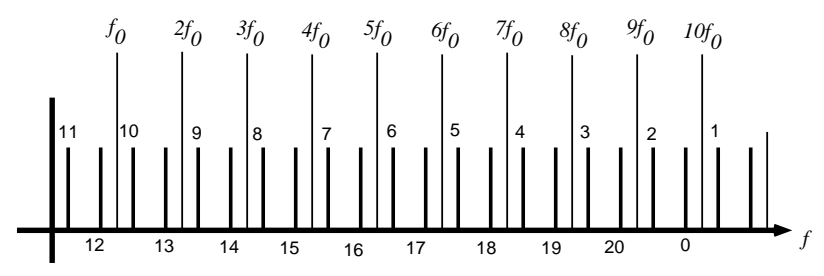

FIG. 3. The coupled-bunch mode spectrum for $M=21$.
Green's function is $G_{m n}(\Omega)=R_{m n}^{-1}(\Omega)$ and it satisfies the equation

$$
\sum_{m=0}^{M-1} R_{n m}(\Omega) G_{m n^{\prime}}(\Omega)=\delta_{n n^{\prime}}
$$

To find it, we expand Green's function using the normal mode eigenvectors as a basis set

$$
\begin{aligned}
G_{m n}(\Omega) & =\frac{1}{M} \sum_{l=0}^{M-1} \sum_{l^{\prime}=0}^{M-1} \exp \left(\frac{2 \pi i m l}{M}\right) \exp \left(\frac{2 \pi i n l^{\prime}}{M}\right) \tilde{G}_{l l^{\prime}}(\Omega), \\
\tilde{G}_{l l^{\prime}}(\Omega) & =\frac{1}{M} \sum_{m=0}^{M-1} \sum_{n=0}^{M-1} \exp \left(-\frac{2 \pi i m l}{M}\right) \exp \left(-\frac{2 \pi i n l^{\prime}}{M}\right) G_{m n}(\Omega)
\end{aligned}
$$

substitute into Eq. (27) and make use of Eq. (11) to get

$$
G_{m n}(\Delta \Omega)=-\frac{1}{2 M \omega_{\beta}} \sum_{l=0}^{M-1} \frac{\exp \left[\frac{2 \pi i l(m-n)}{M}\right]}{\left(\Delta \Omega-\Delta \Omega_{l}\right)},
$$

in which $\Delta \Omega=\Omega-\omega_{\beta}$, and $\Delta \Omega_{l}$ is given by Eq. (19). This result will be used in the following section.

\section{LOCALIZED MODES}

Returning to the original equation of motion, we have, including the octupole nonlinearity,

$$
\frac{d^{2} y_{n}(t)}{d t^{2}}+\omega_{\beta}^{2}\left(a_{n}\right) y_{n}(t)-\sum_{m=0}^{M-1} y_{m}(t) L(m-n)=\frac{d^{2} y_{n}(t)}{d t^{2}}+\omega_{\beta}^{2} y_{n}(t)+\mu^{\prime} a_{n}^{2} y_{n}(t)-\sum_{m=0}^{M-1} y_{m}(t) L(m-n)=0,
$$

where

$$
\mu^{\prime}=2 \mu \omega_{\beta} \omega_{0},
$$

and we assume that $\mu a^{2} \ll \nu$.

We now look for solutions of the form $y_{n}(t)=$ $a_{n} \exp (-i \Omega t)$, in which $a_{n}$ is the oscillation amplitude. Equation (30) becomes

$-\Omega^{2} a_{n}+\omega_{\beta}^{2} a_{n}-\sum_{m=0}^{M-1} a_{m} L(m-n)=-\mu^{\prime} a_{n}^{3}$.

The equation with $\mu^{\prime}=0$ is the same one for which we have found Green's function. Thus, referring to Eq. (26)

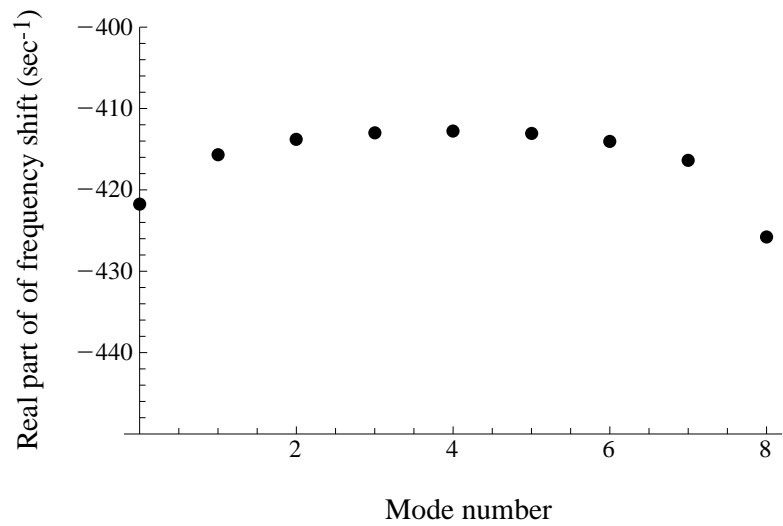

FIG. 4. The real part of $\Delta \Omega_{\mu}$ vs mode number $\mu$, for $M=9$. above, Eq. (32) becomes

$$
\sum_{m=0}^{M-1} R_{n m}(\Omega) a_{m}=-\mu^{\prime} a_{n}^{3},
$$

for which we have the solution, using Green's function,

$$
\begin{aligned}
a_{n} & =-\mu^{\prime} \sum_{m=0}^{M-1} R_{n m}^{-1}(\Omega) a_{m}^{3}=-\mu^{\prime} \sum_{m=0}^{M-1} G_{n m}(\Delta \Omega) a_{m}^{3} \\
& =\frac{\mu^{\prime}}{2 M \omega_{\beta}} \sum_{m=0}^{M-1} \sum_{l=0}^{M-1} \frac{\exp \left[\frac{2 \pi i l(n-m)}{M}\right]}{\left(\Delta \Omega-\Delta \Omega_{l}\right)} a_{m}^{3} .
\end{aligned}
$$

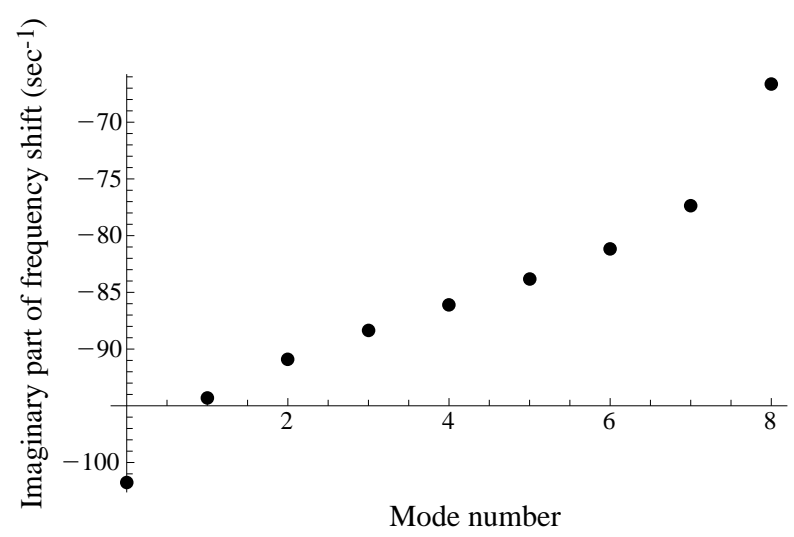

FIG. 5. The imaginary part of $\Delta \Omega_{\mu}$ vs mode number $\mu$, for $M=9$. 


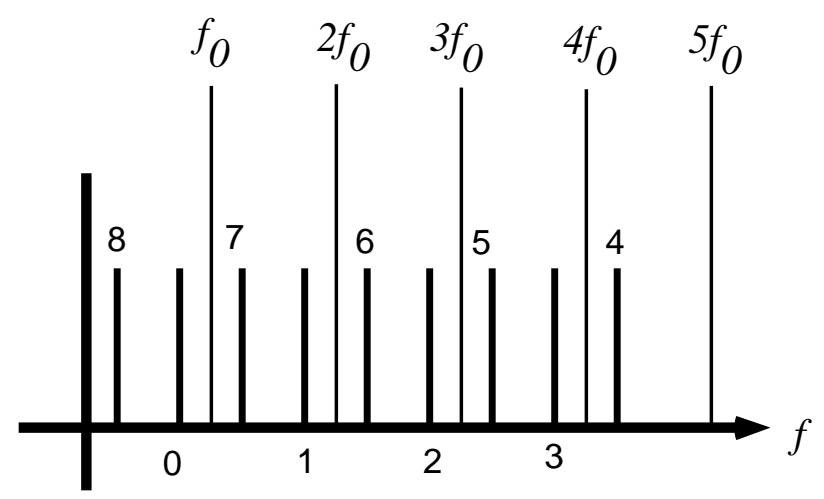

FIG. 6. The coupled-bunch mode spectrum for $M=9$.

Using Eq. (31), we have

$$
a_{n}=\frac{\mu \omega_{0}}{M} \sum_{m=0}^{M-1} \sum_{l=0}^{M-1} \frac{\exp \left[\frac{2 \pi i l(n-m)}{M}\right]}{\left(\Delta \Omega-\Delta \Omega_{l}\right)} a_{m}^{3} .
$$

This is essentially a nonlinear eigenvalue equation. There will be normal modes, corresponding to some linear combination of the $a_{n}$ amplitudes, and for each mode there will be a frequency shift $\Delta \Omega$. Because of the nonlinearity, the mode eigenvector and frequency shift will depend on the amplitude of the oscillation. We are interested in specific modes, which are "localized" to one or two bunches. If $\mu$ is negative, the localized mode will have

a frequency below the lowest normal mode frequency shown in Fig. 1 (which is mode 11 for $M=21$ ). If $\mu$ is positive, the localized mode will have a frequency above the highest normal mode frequency shown in Fig. 1 (which is mode 1 for $M=21$ ).

Following Ref. [5], let $a_{n}=\alpha \xi_{n}$, with $\alpha$ a measure of the oscillation amplitude, and $\max \left(\xi_{n}\right)=1$. Then we have

$$
\xi_{n}=\frac{\mu \alpha^{2} \omega_{0}}{M} \sum_{m=0}^{M-1} \sum_{l=0}^{M-1} \frac{\exp \left[\frac{2 \pi i l(n-m)}{M}\right]}{\left(\Delta \Omega-\Delta \Omega_{l}\right)} \xi_{m}^{3} .
$$

Note that $\mu \alpha^{2} \omega_{0}$ is just the frequency shift produced by the octupole for an oscillation amplitude $\alpha$. Equation (36) can be solved iteratively as follows. We first assume that only the $n=0$ bunch is excited, so $\xi_{0}=1$, and all other $\xi_{n}$ are zero. Then we have

$$
1=\frac{\mu \alpha^{2} \omega_{0}}{M} \sum_{l=0}^{M-1} \frac{1}{\Delta \Omega^{(0)}-\Delta \Omega_{l}},
$$

in which $\Delta \Omega^{(0)}$ is the zero-order eigenfrequency shift. Equation (37) is solved for $\Delta \Omega^{(0)}$, and the result is substituted into Eq. (36) to get

$$
\xi_{1}=\frac{\mu \alpha^{2} \omega_{0}}{M} \sum_{l=0}^{M-1} \frac{\exp \left(\frac{2 \pi i l}{M}\right)}{\Delta \Omega^{(0)}-\Delta \Omega_{l}} .
$$

Then, with this value for $\xi_{1}$, we solve

$$
1=\frac{\mu \alpha^{2} \omega_{0}}{M}\left(\sum_{l=0}^{M-1} \frac{1}{\Delta \Omega^{(1)}-\Delta \Omega_{l}}+\xi_{1}^{3} \sum_{l=0}^{M-1} \frac{\exp \left(-\frac{2 \pi i l}{M}\right)}{\Delta \Omega^{(1)}-\Delta \Omega_{l}}\right),
$$

to get the first-order eigenvalue shift $\Delta \Omega^{(1)}$. This is then used with $\xi_{1}$ to get $\xi_{2}$, and so on. The iterative process converges as long as $\xi_{n} \ll 1$ for $n>0$.

Another approach is to observe that Eq. (36) is a set of $M$ simultaneous nonlinear equations; the $M$ unknowns are the $\xi_{n}$ (except for $\xi_{0}=1$ ) and the frequency shift $\Delta \Omega$. The $M$ equations can then be solved for the $M$ unknowns. This procedure gives the same result as the iteration technique for the example discussed in the next section.

\section{ANALYTICAL RESULTS}

In CESR, there are two octupoles at locations with large vertical beta functions $(23.56 \mathrm{~m}$ and $22.68 \mathrm{~m})$. Each has a length $L=0.391 \mathrm{~m}$. The frequency shift that appears in Eq. (36) can be written as

$$
\begin{aligned}
\Delta \omega_{\text {oct }} & =\mu \alpha^{2} \omega_{0}=\omega_{0} \frac{3}{16 \pi} \frac{k_{3} L}{6} \beta\left(s_{1}\right)^{2} \frac{\alpha^{2}}{\beta_{0}} \\
& =\omega_{0} \frac{3 \varepsilon_{y}}{16 \pi} \frac{k_{3} L}{6} \beta\left(s_{1}\right)^{2}\left(\frac{\alpha}{\sigma_{y}}\right)^{2},
\end{aligned}
$$

in which the rms vertical emittance is $\varepsilon_{y}=\frac{\sigma_{y}^{2}}{\beta_{0}}$ and $\sigma_{y}$ is the rms vertical beam size at the observation point. Using $\omega_{0}=2.45 \times 10^{6} \mathrm{~s}^{-1}$ and $\varepsilon_{y}=3 \times 10^{-9} \mathrm{~m} \mathrm{rad}$, we get, summing over the two octupoles,

$$
\Delta \omega_{\text {oct }}=0.0306 k_{3}\left[\mathrm{~m}^{-4}\right]\left(\frac{\alpha}{\sigma_{y}}\right)^{2} \mathrm{~s}^{-1} .
$$

For example, for $k_{3}=-49 \mathrm{~m}^{-4}$ in both octupoles and $\alpha=8 \sigma_{y} \quad(2 \mathrm{~mm}$ at $\beta=20 \mathrm{~m})$, we have $\Delta \omega_{\text {oct }}=$ $-96 \mathrm{~s}^{-1}$.

With this nonlinearity, the solution of Eq. (36) for $M=21$ gives a localized mode with a frequency shift of $-504.9-85.6 i \mathrm{~s}^{-1}$. It is shifted down by about $63 \mathrm{~s}^{-1}$ from the linear coupled-bunch mode 11 frequency. Figure 7 plots $\xi_{n}$, for $n=0,1, \ldots, 20$, around the ring; the distance from the dot to the circle is proportional to the oscillation amplitude of each bunch. The largest value corresponds to $\xi_{0} ; n$ increases in the clockwise direction. The maximum amplitude is about $2 \mathrm{~mm}$ at $\beta=20 \mathrm{~m}$.

For $k_{3}=49 \mathrm{~m}^{-4}$ units in both octupoles and $\alpha=$ $8 \sigma_{y}$, we have $\Delta \omega_{\text {oct }}=96 \mathrm{~s}^{-1}$. With this nonlinearity, the solution of Eq. (36) with $M=21$ gives a localized mode with a frequency shift of $-317.7-86.6 i \mathrm{~s}^{-1}$. It is shifted up by about $89 \mathrm{~s}^{-1}$ from the linear coupled-bunch mode 1 frequency. The bunch pattern corresponding to this localized mode is shown in Fig. 8.

For $M=9, \Delta \omega_{\text {oct }}=-96 \mathrm{~s}^{-1}$ gives a frequency shift of $-511.3-85.3 i \mathrm{~s}^{-1}$ (shifted down by $85.5 \mathrm{~s}^{-1}$ from 


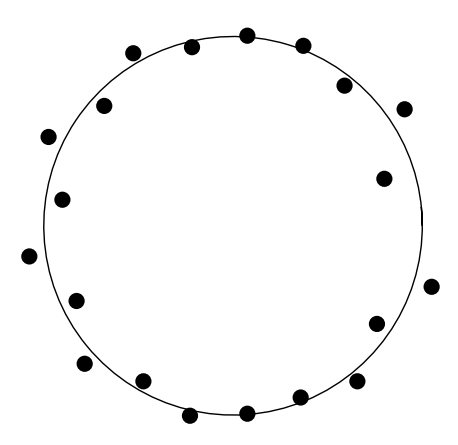

FIG. 7. The localized mode bunch pattern for $\Delta \omega_{\text {oct }}=$ $-96 \mathrm{~s}^{-1}$ and $M=21$.

linear coupled-bunch mode 8); the localized mode pattern is shown in Fig. 9.

For $M=9$ and $\Delta \omega_{\text {oct }}=96 \mathrm{~s}^{-1}$, we get a frequency shift of $-320.9-85.9 i \mathrm{~s}^{-1}$ (shifted up by $91.9 \mathrm{~s}^{-1}$ from linear coupled-bunch mode 4). The localized mode pattern is shown in Fig. 10.

\section{NUMERICAL SIMULATION RESULTS}

To provide a confirmation of the approximations made in the analytical approach, a numerical simulation has been performed. This simulation starts with a given set of initial conditions $\mathbf{y}_{0}, \dot{\mathbf{y}}_{0}$. The corresponding initial values of the normal modes are given from Eq. (6) by $\mathbf{q}_{0}=\overleftrightarrow{\mathbf{C}} \cdot \mathbf{y}_{0}, \dot{\mathbf{q}}_{0}=\overleftrightarrow{\mathbf{C}} \cdot \dot{\mathbf{y}}_{0}$. The evolution of the normal modes over one turn is, from Eq. (9),

$$
\begin{aligned}
& q_{i}\left(T_{0}\right)=q_{i 0} \cos \left(\Omega_{i} T_{0}\right)+\frac{\dot{q}_{i 0}}{\Omega_{i}} \sin \left(\Omega_{i} T_{0}\right), \\
& \dot{q}_{i}\left(T_{0}\right)=\dot{q}_{i 0} \cos \left(\Omega_{i} T_{0}\right)-\Omega_{i} q_{i 0} \sin \left(\Omega_{i} T_{0}\right),
\end{aligned}
$$

in which $\Omega_{i}=\omega_{\beta}+\Delta \Omega_{i}$, with $\Delta \Omega_{i}$ from Eq. (19). (For simplicity, the imaginary part of the frequency shift has been neglected.) The values of $\mathbf{y}, \dot{\mathbf{y}}$ after one turn

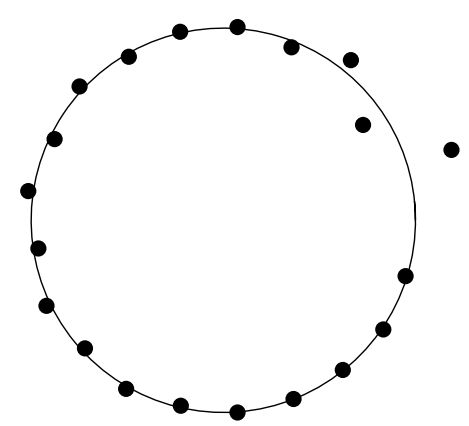

FIG. 8. The localized mode bunch pattern for $\Delta \omega_{\text {oct }}=$ $96 \mathrm{~s}^{-1}$ and $M=21$.

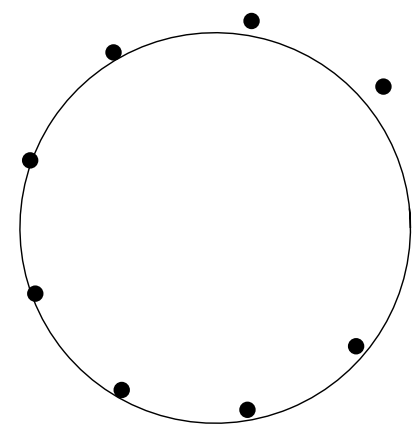

FIG. 9. The localized mode bunch pattern for $\Delta \omega_{\text {oct }}=$ $-96 \mathrm{~s}^{-1}$ and $M=9$.

are then calculated from $\mathbf{y}\left(T_{0}\right)=\overleftrightarrow{\mathbf{C}}^{-1} \cdot \mathbf{q}\left(T_{0}\right), \dot{\mathbf{y}}\left(T_{0}\right)=$ $\overleftrightarrow{\mathbf{C}}^{-1} \cdot \dot{\mathbf{q}}\left(T_{0}\right)$. The effect of the octupole is to produce a kick on each turn given by

$$
\dot{y}_{i}\left(T_{0}\right) \rightarrow \dot{y}_{i}\left(T_{0}\right)-\frac{c k_{3}}{6}\left[y_{i}\left(T_{0}\right)\right]^{3} .
$$

The resulting values of $\mathbf{y}\left(T_{0}\right), \dot{\mathbf{y}}\left(T_{0}\right)$ are used as initial conditions to find initial normal mode values to start the next turn. The process is repeated for as many turns as required.

The results are shown in Figs. 11 and 12, for $M=9$, and for the other parameters as given in Sec. VIII above (with $k_{3}>0$ ). In each figure, the maximum values of $y_{i}$ during a betatron oscillation are shown, for each of the nine bunches, for 200000 turns. The initial conditions in each case have $y_{i 0}=0$ for all $i$ except $i=1$, for which $y_{10}=2.5 \mathrm{~mm}$, and $\dot{y}_{i 0}=0$ for all $i$.

Figure 11 corresponds to the linear case: As the normal modes evolve, bunches other than bunch 1 develop some oscillation amplitude, due to the dispersion in the system. Figure 12 is the result obtained with the nonlinear term included. In this case, the oscillation remains localized on the original bunch, confirming the presence of the localized mode as predicted by the analytical results.

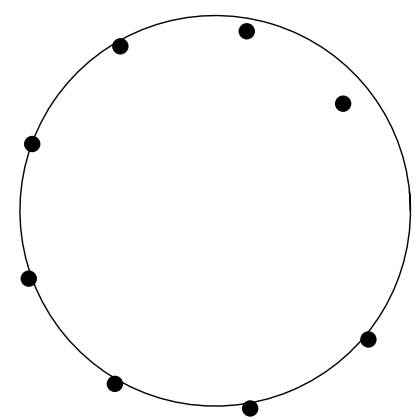

FIG. 10. The localized mode bunch pattern for $\Delta \omega_{\text {oct }}=$ $96 \mathrm{~s}^{-1}$ and $M=9$. 

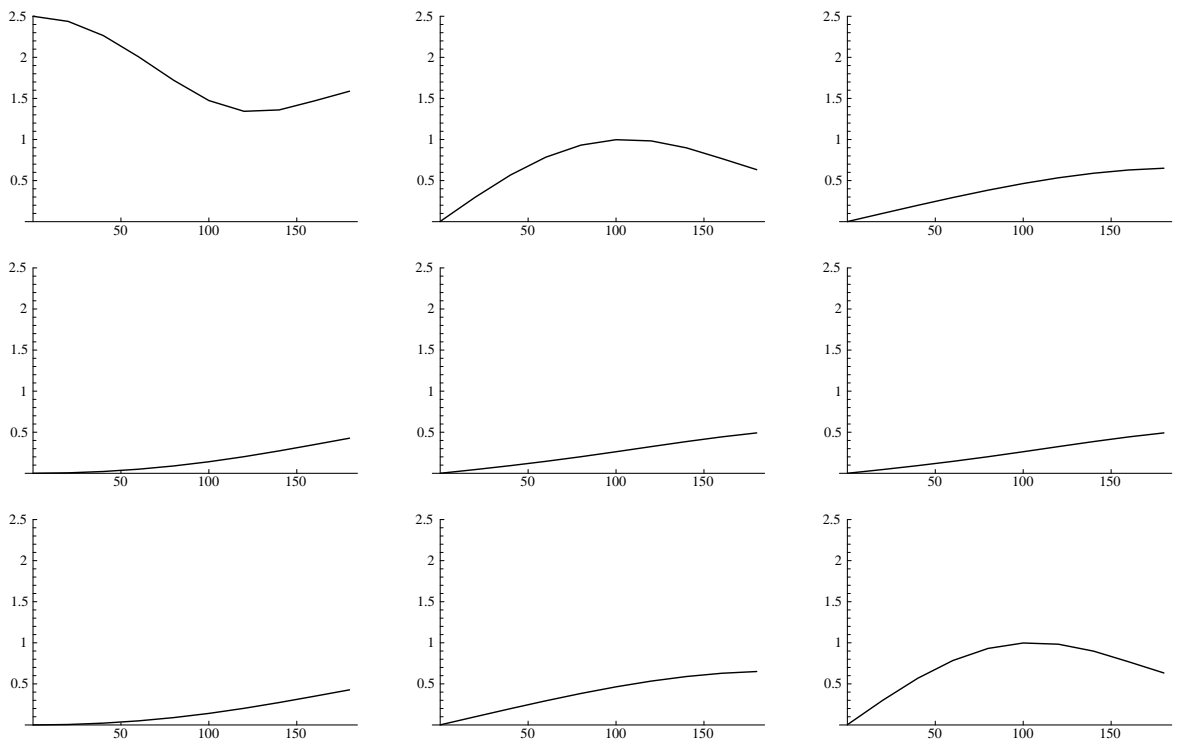

FIG. 11. The evolution of the linear normal modes. The plots show the maximum amplitude of the oscillation (in mm) vs turn number (in thousands of turns), for bunches 1 to 9 (left to right, from top left to bottom right).
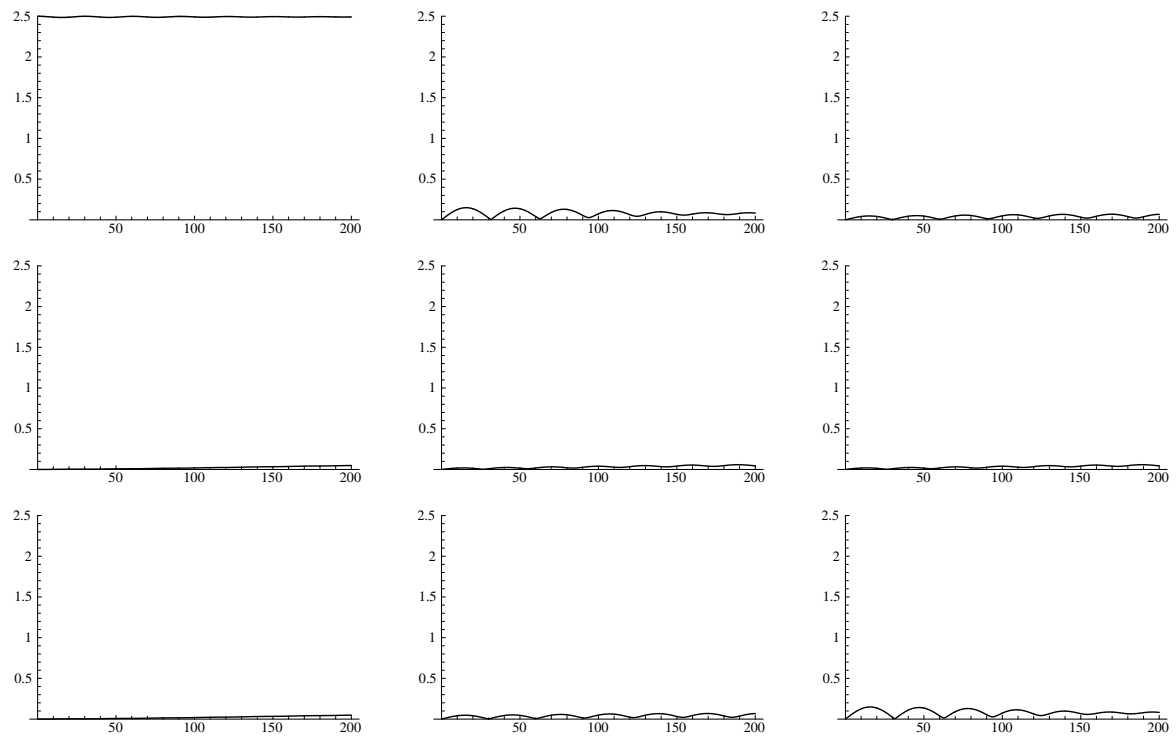

FIG. 12. The evolution of the localized mode. The plots show the maximum amplitude of the oscillation (in mm) vs turn number (in thousands of turns), for bunches 1 to 9 (left to right, from top left to bottom right).

\section{CONCLUSION}

Localized modes may be possible in the coherent motion of arrays of bunches in CESR, in the presence of nonlinearities generated by octupoles. With a bunch-to-bunch coupling produced by the resistive wall impedance, with vertical oscillation amplitudes of about $2 \mathrm{~mm}$ (at $\beta_{y}=$ $20 \mathrm{~m}$ ), and for octupole strengths of about $50 \mathrm{~m}^{-4}$, these modes appear for both 21 and 9 equally spaced, equally populated bunches of $1.3 \times 10^{11}$ electrons per bunch.

\section{ACKNOWLEDGMENTS}

The author wishes to thank A. Sievers for suggesting the possible existence of these modes in accelerators and for many helpful discussions. Additional help has been 
provided by J.T. Rogers, who modeled some of these effects in a spreadsheet program. This work was supported by the National Science Foundation.

[1] A. Sievers (private communication).

[2] J.T. Rogers (private communication).

[3] S. Takeno, Prog. Theor. Phys. 75, 1 (1986).

[4] A. J. Sievers and S. Takeno, Phys. Rev. Lett. 61, 8 (1988).

[5] S. Takeno, K. Kisoda, and A. Sievers, Prog. Theor. Phys.
Suppl. 94, 1 (1988).

[6] J. B. Page, Phys. Rev. B 41, 11 (1988).

[7] S. R. Bickham, S. A. Kislev, and A.J. Sievers, in Spectroscopy and Dynamics of Collective Excitations in Solids, edited by B. DiBartolo (Plenum, New York, 1997).

[8] A. Chao, Physics of Collective Beam Instabilities in High Energy Accelerators (Wiley, New York, 1993), p. 205.

[9] A. Chao, Physics of Collective Beam Instabilities in High Energy Accelerators (Ref. [8]), p. 345.

[10] A. Chao, Physics of Collective Beam Instabilities in High Energy Accelerators (Ref. [8]), p. 71. 